\title{
SYNERGIES BETWEEN ELECTROMAGNETIC CALORIMETRY AND PET $^{*}$
}

\author{
W. W. MOSES
}

Lawrence Berkeley National Laboratory

Mailstop 55-121, 1 Cyclotron Road

Berkeley, CA 94709, USA

E-mail:wwmoses@lbl.gov

\begin{abstract}
The instrumentation used for the nuclear medical imaging technique of Positron Emission Tomography (PET) shares many features with the instrumentation used for electromagnetic calorimetry. Both fields can certainly benefit from technical advances in many common areas, and this paper discusses both the commonalties and the differences between the instrumentation needs for the two fields. The overall aim is to identify where synergistic development opportunities exist. While such opportunities exist in inorganic scintillators, photodetectors, amplification and readout electronics, and high-speed computing, it is important to recognize that while the requirements of the two fields are similar, they are not identical, and so it is unlikely that advances specific to one field can be transferred without modification to the other.
\end{abstract}

\section{Introduction}

There are many similarities between the instrumentation for electromagnetic calorimetry and PET. Incident gamma rays are detected (and their energy and arrival time measured) with inorganic scintillators coupled to photodetectors. Thousands of these channels are read out in parallel using custom analog integrated circuits. However, each field has its own unique requirements, which often force different optimizations to be made. This paper explores the similarities and differences between the fields. It assumes that the reader is familiar with high energy physics and electromagnetic calorimetry, but relatively unfamiliar with PET.

\section{The PET World Picture}

PET is a nuclear medical imaging technique whereby the patient is injected with positron-labeled radiopharmaceutical ${ }^{1,2,3,4,5}$. This drug localizes within the patient according to its physiologic properties and the radioisotope decays, emitting a positron. The positron annihilates with an electron (from the patient's tissue) to

* This work was supported in part by the U.S. Department of Energy under contract No. DE-AC0376SF00098, and in part by Public Health Service Grants Nos P01-HL25840 and R01-CA67911. 
form back-to-back $511 \mathrm{keV}$ annihilation photons. As shown in Figure 1, a planar ring of $511 \mathrm{keV}$ photon detectors surround the patient - if two photons are detected simultaneously (within $10 \mathrm{~ns}$ ) then the radioisotope is assumed to lie somewhere along the line joining the two detector elements. The mathematical technique of computed tomography ${ }^{6}$, shown in Figure 2, is used to reconstruct the two-dimensional activity distribution within the plane defined by that detector ring - images from multiple detector rings are used to create a three-dimensional, volumetric image of the radiopharmaceutical distribution. PET's strength is that it images the physiological properties of the pharmaceutical and so provides physiologic information, whereas many other medical imaging techniques provide mainly anatomical information ${ }^{7}$. For example, $x$-ray techniques image electron density and MRI (magnetic resonance imaging) mainly images water density. Therefore PET is predominantly used for diseases that are metabolically based (such as cancer or neurological disease).

Despite the many similarities between PET and electromagnetic calorimetry, there are significant differences. One of the main constraints in PET is the low energy of the photons that must be measured with high accuracy $-511 \mathrm{keV}$ is many orders of magnitude lower than the energies commonly measured with calorimeters, implying greater signal-to-noise challenges in PET. This also implies that there are no electromagnetic showers involved in PET - the only relevant interaction processes are photoelectric absorption and Compton scatter, and Compton scatter is the dominant process (at $511 \mathrm{keV}$, the photoelectric fraction is $45 \%$ in BGO scintillator and $0.02 \%$ for tissue). The high Compton fraction and relatively short interaction length $(10 \mathrm{~cm})$ in tissue also imply that in only a small percentage $(<10 \%)$ of the positron annihilations do both $511 \mathrm{keV}$ photons exit the patient without undergoing Compton scatter.

Most PET studies are limited by counting statistics, as it is generally impossible to increase the imaging time (due to the half-life of the radioisotope and the requirement that the patient remain motionless for the up to two hour duration of the study) or the injected dose (there are limits to the radiation dose that the patient may receive). There is, however, a silver lining to the dosage limitation - PET instrumentation does not have the significant radiation damage constraints that are placed on calorimetry.

The detectors in most PET cameras consist of block of BGO scintillator crystal that is partially sawn through to make a group of quasi-independent crystals that are optically coupled to four photomultiplier tubes, as shown in Figure 3. When a gamma ray interacts in the crystal, the resulting scintillation photons are emitted isotropically but the saw cuts limit (but do not entirely prevent) their lateral dispersion as they travel toward the photomultiplier tubes. The position of the gamma ray interaction within the scintillator block is then determined by the analog ratio of the photomultiplier tube output signals, and the gamma ray energy is determined and a timing pulse generated by the sum of these signals ${ }^{8}$. Based on current PET cameras, the gamma ray detector requirements for PET are, in order of decreasing importance, (1) $>85 \%$ 

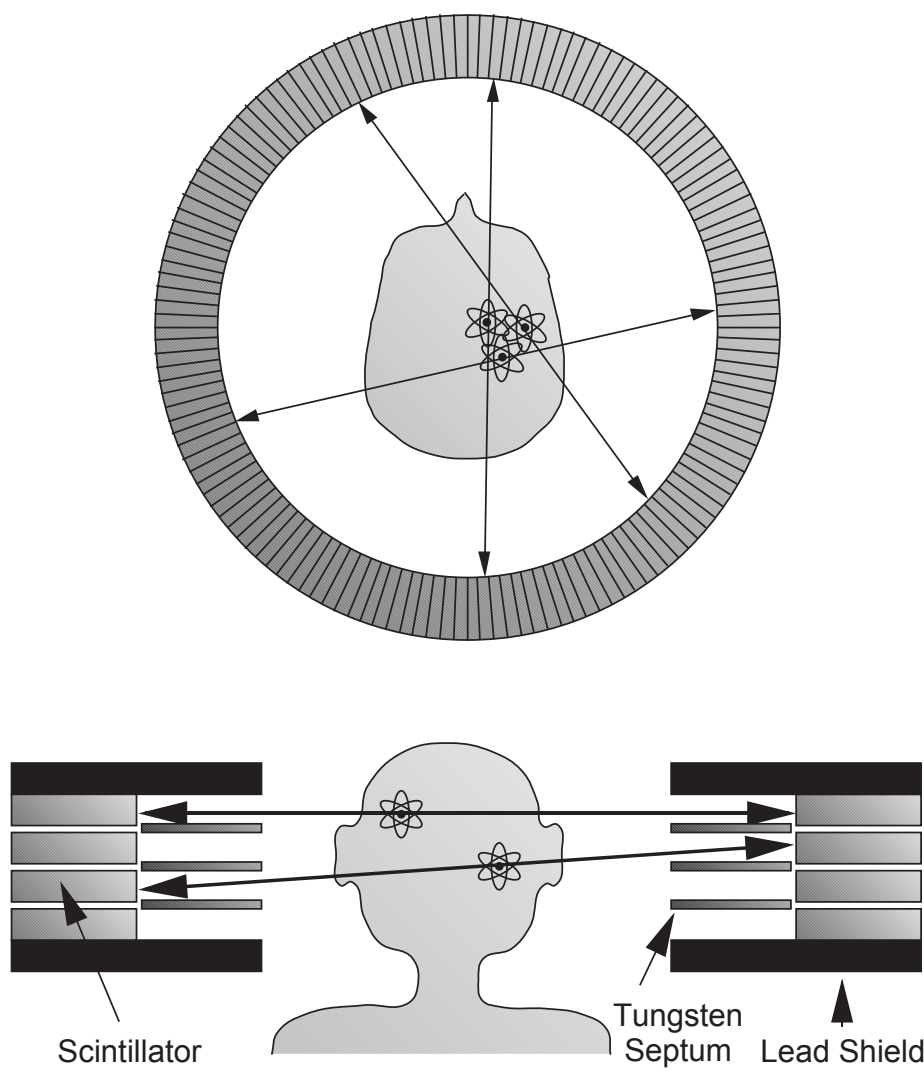

Figure 1. PET Camera Schematic. Positron annihilations yield back to back $511 \mathrm{keV}$ photons, which are individually detected in a ring of photon detectors, shown on the top. Pairs are identified by time coincidence. Multiple rings are stacked up, as shown on the bottom, to create a 3-dimensional image.

detection efficiency (to minimize statistical fluctuations), (2) $<5 \mathrm{~mm}$ fwhm position resolution (to obtain good spatial resolution), $(3)<\$ 100 / \mathrm{cm}^{2}$ parts cost (PET cameras are widely available commercially), (4) $<1 \mu \mathrm{s} \mathrm{cm}^{2}$ dead time product (single event dead time times detector area affected - high counting rates are often encountered), (5) $<2 \mathrm{~ns}$ timing resolution (to identify coincident pairs), and (6) $<100 \mathrm{keV}$ fwhm energy resolution (to reject Compton scatter events) ${ }^{9}$.

Finally, the competitive commercial market for PET cameras limits their cost. The CMS electromagnetic calorimeter has 72,000 channels and an approximate parts cost of $\$ 60$ million, implying a parts cost of $\$ 833$ per channel. A modern PET camera, on the other hand, contains approximately one quarter of the channels $(18,400)$ but nearly two orders of magnitude lower parts cost (\$1 million), implying a parts cost of only $\$ 54$ per channel. Of the total parts cost for a PET camera, approximately 


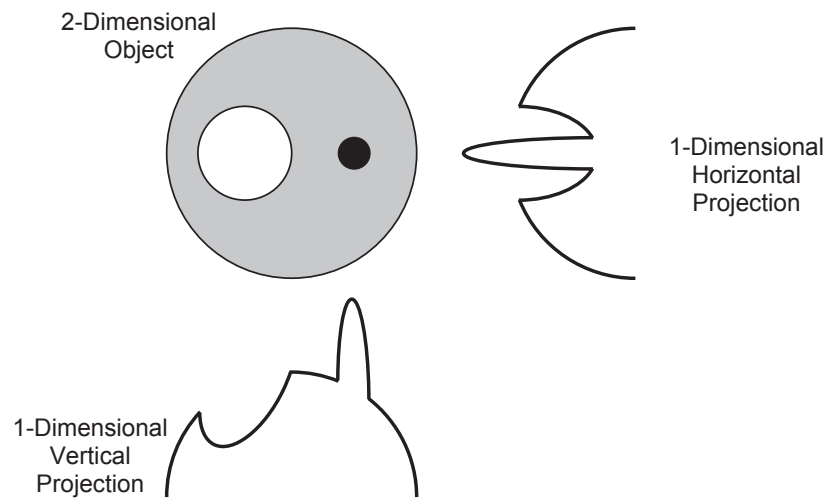

Figure 2. Computed Tomography. The 1-dimensional horizontal and vertical projections (the line integral of the density along parallel horizontal and vertical lines) are shown adjacent to the 2-dimensional object that they were taken of. Computed tomography is the process of reconstructing the 2-dimensional object given its 1-dimensional projections from all angles.

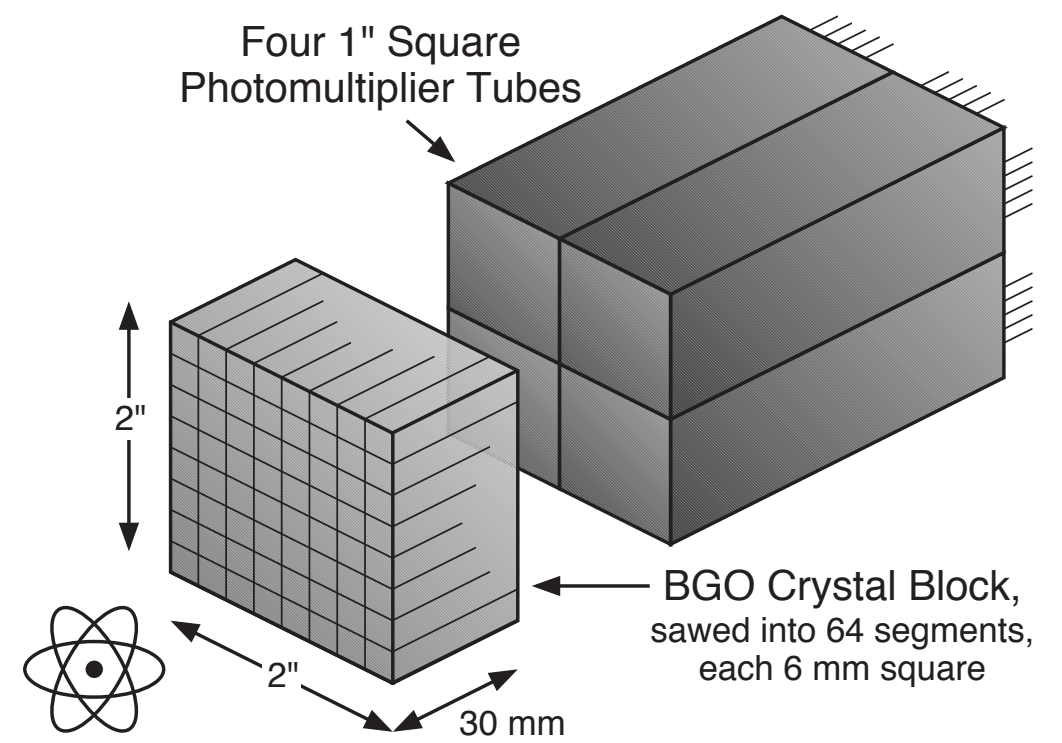

Figure 3. PET Detector Module. Scintillation light from gamma ray interactions is detected by multiple photomultiplier tubes. The interaction position is determined by the ratio of the analog signals, and the energy by the analog sum of the signals.

$25 \%$ is scintillator crystal, $25 \%$ is photomultiplier tubes, and no other component represents more than $10 \%$ of the parts cost. Thus, cost must be carefully considered when evaluating replacement detector technologies. 


\section{Comparison of Calorimetry and PET}

\subsection{Scintillators}

For both electromagnetic calorimetry and PET, the highest performance instruments use inorganic scintillator crystals coupled to photodetectors to detect gamma rays with high efficiency, energy resolution, spatial resolution, and timing resolution. The last decade has seen remarkably parallel developments of a new scintillator for electromagnetic calorimetry $\left(\mathrm{PbWO}_{4}\right)^{10,11}$ and a new scintillator for PET (LSO) ${ }^{12}$. Both were discovered in approximately 1992, both are just now entering the full-scale production phase, and the development of both have required the efforts not only of the end users, but also of luminescence scientists, spectroscopists, defects scientists, materials scientists, and crystal growers.

While an ideal scintillator would satisfy the requirements for both disciplines, the ideal scintillator has not yet been developed and compromises must be made. As Table 1 indicates, many properties of these two newly developed scintillators are very similar, such as the density, attenuation length, decay time, and emission wavelength. However, there are some important differences (which are highlighted in bold face type). The light output of LSO is much higher than $\mathrm{PbWO}_{4}$ in order to (partially) compensate for the relatively low energy of the photons measured by PET. The cost per unit volume of $\mathrm{PbWO}_{4}$ scintillator must be significantly lower than scintillators used for PET - even though the budget per channel is almost two orders of magnitude higher for calorimetry than it is for PET, the volume of scintillator required for PET is roughly three orders of magnitude higher. Finally, $\mathrm{PbWO}_{4}$ must be very radiation hard in order to withstand the background radiation present at high luminosity hadron colliders. Although LSO happens to be fairly radiation hard (10 Mrads), it could be several orders of magnitude less radiation hard without impairing its performance for PET.

Table 1. Scintillator properties for $\mathrm{PbWO}_{4}$ and LSO scintillator.

\begin{tabular}{|l|c|c|}
\hline & $\mathrm{PbWO}_{4}$ & LSO \\
\hline Density (g/cc) & 8.3 & 7.4 \\
Attenuation Length (cm) & 0.9 & 1.2 \\
Light Output (photons/MeV) & $\mathbf{2 0 0}$ & $\mathbf{2 5 , 0 0 0}$ \\
Decay Time (ns) & 10 & 40 \\
Emission Wavelength (nm) & 420 & 420 \\
Radiation Hardness (Mrad) & $>\mathbf{1 0}$ & $\mathbf{1 0}$ \\
Dopants & $\mathrm{Y}, \mathrm{Nb}$ & $\mathrm{Ce}$ \\
Cost per cc & $\mathbf{\$ 1 . 6}$ & $>\mathbf{\$ 2 5}$ \\
\hline
\end{tabular}




\subsection{Avalanche Photodiodes}

Avalanche photodiode (APD) arrays promise substantial improvements for PET $^{9}$. They promise a pixel size that is much better matched to that of the scintillator crystals (3-5 mm) with minimal dead area between pixels, which leads to higher spatial resolution. APD arrays are not without challenges though. For PET, minimal dead area around the perimeter of the packaged device is essential, and large (or even moderate) scale manufacturing techniques have not yet matured to where the cost and reliability cease to be issues. Finally, while their high quantum efficiency and gain promise to give acceptable system energy resolution and timing resolution, their signal to noise ratio is likely to be inferior to that of PMT-based systems.

Many of these same features have made APD arrays attractive to the electromagnetic calorimeter community, which has worked extensively to develop these devices. As with the scintillator material, the optimization of tradeoffs is different for high energy physics than it is for PET, and is summarized in Table 2. While both applications desire high gain and high quantum efficiency (especially at the shorter wavelength region of the visible spectrum), high energy physics experiments have a high flux of charged particles traversing the APDs. This requires that the APDs be radiation hard as well as have a relatively thin (typically $100 \mu \mathrm{m}$ ) depletion depth to minimize the nuclear counter effect. APDs used for PET do not have these restrictions, but have tighter signal-to-noise requirements. In order to obtain low electronic noise at high frequencies (to obtain an accurate timing signal), the depletion region for PET APDs should be as large as possible in order to minimize the detector capacitance. Leakage current must also be minimized for both applications, but the requirements are more stringent for PET. Finally, the pixels in PET are roughly an order of magnitude smaller than they are for calorimetry. While neither application can tolerate significant gaps between pixels (i.e., high packing fraction is needed for both), the smaller size of the pixels in PET implies that the size of the inactive area around the perimeter of the array must be proportionally smaller for PET.

\subsection{Electronics}

The electronics for electromagnetic calorimetry and PET share many features. Large numbers of analog amplifiers with very low noise and low power consumption are required, and are usually implemented in mixed-mode (analog and digital) custom ASICs. Reasonably complex data correction is performed on-the-fly. The data acquisition systems support extremely high data rates, generally by using highly parallel architectures.

However, there are also many differences in the electronics, and these are summarized in Table 3. As with the scintillator and APDs, radiation hardness is critical for calorimetry but irrelevant for PET. The analog electronics for calorimetry must 
Table 2. Avalanche photodiode requirements for electromagnetic calorimetry and PET.

\begin{tabular}{|l|c|c|}
\hline & Calorimetry & PET \\
\hline High Gain? & Yes & Yes \\
High QE / Blue Sensitivity? & Yes & Yes \\
Radiation Hardness? & High & Low \\
Reduced Nuclear Counter Effect? & Yes & No \\
Timing Signal (low C)? & No & Yes \\
Small Dead Area at Perimeter? & No & Yes \\
Sensitive to Leakage Current? & - & Yes \\
\hline
\end{tabular}

Table 3. Electronics requirements for electromagnetic calorimetry and PET.

\begin{tabular}{|l|c|c|}
\hline & Calorimetry & PET \\
\hline Low Noise Analog Amplifier? & Yes & Yes \\
Low Power Consumption? & Yes & Yes \\
Mixed-Mode Custom ASICs? & Yes & Yes \\
Real-Time Data Correction? & Yes & Yes \\
Highly Parallel readout? & Yes & Yes \\
High Data Rate? & Yes & Yes \\
& & \\
Radiation Hardness? & Yes & No \\
Analog Dynamic Range? & High & Low \\
Self-Generated Timing Signal? & No & Yes \\
Asynchronous Inputs? & No & Yes \\
Event Size / Complexity? & High & Low \\
Multiple Trigger Levels? & No & Yes \\
"Good" Event Rate? & kHz & MHz \\
\hline
\end{tabular}

accurately measure input signals that span a large dynamic range, but the dynamic range for PET is low, as the input signals consist mainly of monochromatic, $511 \mathrm{keV}$ photons (the remainder are $511 \mathrm{keV}$ photons that have undergone Compton scatter). The clocking / synchronization of the systems are also quite different. For high energy physics, events can only be generated when the accelerator bunches cross, and the accelerator provides the readout electronics a timing signal that is synchronized to this bunch crossing. Thus, the electronics for high energy physics are externally clocked with a constant clock period. With PET, events are produced when radioiso- 
topes undergo radioactive decay, which is an excellent physical example of a random process. Finally, there are major differences in the event data that must be collected. With high energy physics, many different event topologies are possible, implying that complicated, multi-level event triggering schemes are required and that the size of the event (number of bits that are transferred) is highly variable. However, the overall event rate is relatively low. With PET, only a single event topology is possible (a pair of detected $511 \mathrm{keV}$ gamma rays), leading to simple triggering schemes and a fixed event size. However, the overall event rate is comparatively high.

\subsection{Computing}

PET and electromagnetic calorimetry share many computing requirements, which are summarized in Table 4. In both cases a significant amount of computation is needed, both before and after the apparatus is constructed. Extensive Monte Carlo simulations are performed at the design stage, modeling both the interactions of gamma radiation in the detectors and the underlying physics that will be measured. Both are reasonably large software projects, necessitating many programmers working in a well-coordinated way.

However, there are also many differences. The size of data set for a high energy physics experiment is enormous, possibly ranging from terabytes to petabytes. In contrast, the data set size for a single PET experiment is measured in megabytes to gigabytes. The complexity of the analysis for high energy physics is quite high, with many different experimental signatures that must be recognized. Although the same data is mined many times, the analysis for each "experiment" (i.e. an analysis of the data set resulting in the measurement of a single physics result, often synonymous with "Ph.D. thesis") is different and custom code must be developed for each of these experiments. Although the images produced by PET may be complex, the data from each "experiment" (in this case, one patient study) is reconstructed the same way using the same code. However, the time available to analyze the data from PET is only a few minutes, as imaging centers need to verify that good quality images have been obtained before the patient can be released. High energy physics analyses, on the other hand, can often take years to complete. Finally, software bugs in PET can lead to incorrect diagnosis with potentially fatal results! Because of this, a significantly higher level of scrutiny and approval by the Food and Drug Administration (FDA) is required for PET software.

\section{Discussion}

It is clear that PET and electromagnetic calorimetry have much in common. At the most basic level, the requirements (such as highly efficient detection of gamma rays) and core technologies are identical. However, the previous section makes it evident 
Table 4. Computing requirements for electromagnetic calorimetry and PET.

\begin{tabular}{|l|c|c|}
\hline & Calorimetry & PET \\
\hline Significant Computation? & Yes & Yes \\
Monte Carlo Simulation? & Yes & Yes \\
Large Programming Project? & Yes & Yes \\
Data Set Size? & TB-PB & MB-GB \\
Complexity of Analysis? & High & Low \\
Time to Finish Analysis? & Years & Minutes \\
FDA Certification Required? & No & Yes \\
\hline
\end{tabular}

that the two disciplines have different requirements in a number of areas, some of which are contradictory. These differences imply that no matter how attractive it may be, it is naive to expect that technologies or devices developed and optimized for electromagnetic calorimetry can be transferred unchanged to PET. Does this suggest that synergies between calorimetry and PET are impossible? Far from it! It merely means that effort is needed to translate technologies optimized for one discipline to the other discipline. The high energy physics community has traditionally been at the forefront of developing advanced tools and technologies. The nuclear medical imaging community often adapts the tools and technologies most relevant to its needs, optimizing them to satisfy the unique requirements and tradeoffs required for PET imaging. This transfer is beneficial to both communities and should continue to be encouraged. As is common in such situations, the most effective element in nurturing this synergy is probably communication between the communities, most especially in understanding the requirements and tradeoffs involved in the application-specific optimization.

\section{Conclusion}

Electromagnetic calorimetry and PET both rely on highly efficient detection of gamma rays, hence the technologies used by these two disciplines are extremely similar and many of the tools and technologies currently under development could be applied to either discipline. Examples of these tools and technologies include scintillators, photodetectors (especially APD arrays), electronics, and high-performance computation. The resulting devices are extremely similar - the differences lie mostly in the details. However, the details are important and often preclude having a device that is developed and optimized for one application from being used without modification by the other. Nevertheless, strong synergies exist and should be encouraged, as the effort required to make a modification is often less than that necessary for the 
initial creation.

\section{Acknowledgments}

This work was supported in part by the Director, Office of Science, Office of Biological and Environmental Research, Medical Science Division of the U.S. Department of Energy under Contract No. DE-AC03-76SF00098, and in part by the National Institutes of Health, National Cancer Institute under grant No. R01-CA67911, and National Institutes of Health, National Heart, Lung, and Blood Institute under grant No. P01-HL25840.

\section{References}

1. Sandler, M.P., Coleman, R.E., Wackers, F.J.T., et al., Diagnostic Nuclear Medicine, Baltimore, MD: Williams \& Wilkins (1996).

2. Hendee, W.R. and Ritenour, R., Medical Imaging Physics, St. Louis, MO: Mosby Year Book (1992)

3. Macovski, A., Medical Imaging Systems, Englewood Cliffs, NJ: Prentice Hall (1983).

4. Webb, S., The Physics of Medical Imaging, Bristol: Institute of Physics Publishing (1993).

5. Cherry, S.R. and Phelps, M.E., Positron Emission Tomography: Methods and Instrumentation, In Diagnostic Nuclear Medicine, (Edited by Sandler, M.P., Coleman, R.E., Wackers, F.J.T., Patton, J.A., Gottschalk, A. and Hoffer, P.B.), Baltimore, MD: Williams \& Wilkins, 139 (1996).

6. Cormack, A.M. J. Appl. Phys. 34, 2722-2727 (1963).

7. J. Nucl. Med. 32, 561-748 (1991).

8. Cherry, S.R., Tornai, M.P., Levin, C.S., et al. IEEE Trans. Nucl. Sci. NS-42, 1064 (1995).

9. Moses, W.W., Derenzo, S.E. and Budinger, T.F. Nucl. Instr. Meth. A-353, 189 (1994).

10. P. Lecoq and M. Korzhik, IEEE Trans. Nucl. Sci. NS-47, 1311 (2000).

11. Qu, X., Zhang, L., Zhu, R.Y., et al. Nucl. Instr. Meth. A-480, 470 (2002).

12. C.L. Melcher and J.S. Schweitzer, IEEE Trans. Nucl. Sci. NS-39, 502 (1992). 